\title{
INTERPRETATION OF THE $p$-ADIC LOG GAMMA FUNCTION AND EULER CONSTANTS USING THE BERNOULLI MEASURE \\ BY
}

NEAL KOBLITZ

\begin{abstract}
A regularized version of J. Diamond's $p$-adic log gamma function and his $p$-adic Euler constants are represented as integrals using B. Mazur's $p$-adic Bernoulli measure.
\end{abstract}

1. Introduction. Let $\mathbf{Z}_{p}, \mathbf{Z}_{p}^{x}, \Omega_{p}$ denote, respectively, the $p$-adic integers, the $p$-adic integers not divisible by $p$, and the completion of the algebraic closure of the field of $p$-adic numbers. Let ||$_{p}$ be the absolute value on $\Omega_{p}$ with $|p|_{p}=p^{-1}$.

J. Diamond [3] defined a $p$-adic log gamma function

$$
G_{p}(x)=\lim _{k \rightarrow \infty} p^{-k} \sum_{0<n<p^{k}}(x+n) \log _{p}(x+n)-(x+n) \text { for } x \in \Omega_{p}-\mathbf{Z}_{p}
$$

and a closely related function

$$
\begin{aligned}
G_{p}^{*}(x) & =\lim _{k \rightarrow \infty} p^{-k} \sum_{0<n<p^{k}, p \nmid n}(x+n) \log _{p}(x+n)-(x+n) \\
& =G_{p}(x)-G_{p}(x / p) \quad \text { for } x \in \Omega_{p}-\mathbf{Z}_{p} .
\end{aligned}
$$

Our purpose is to define a "regularized" version of $G_{p}^{*}$ and show that it can be represented as a simple integral over $\mathbf{Z}_{p}^{x}$ with respect to Mazur's $p$-adic Bernoulli measure $\mu_{\alpha}$, namely, as the "convolution" of $\mu_{\alpha}$ with the $p$-adic logarithm (see (7) below). Recall (see [9], or Chapter II of [6]) that for $\alpha \in 1+p \mathbf{Z}, \alpha \neq 1, \mu_{\alpha}$ is defined by

$$
\mu_{\alpha}\left(a+p^{m} \mathbf{Z}_{p}\right)=\alpha^{-1}\left[\alpha a p^{-m}\right]+\left(\alpha^{-1}-1\right) / 2 .
$$

(Actually, any $\alpha \in \mathbf{Z}_{p}^{x}$ not a root of 1 can be used to regularize; but we shall take $\alpha \in 1+p \mathbf{Z}$ for simplicity.) The moments of $\mu_{\alpha}$ give the $p$-adic zeta-function $\zeta_{p}$ that was first defined by Kubota and Leopoldt [8].

Received by the editors April 8, 1977.

AMS (MOS) subject classifications (1970). Primary 12B40; Secondary 33A15.

Key words and phrases. Log gamma function, Euler constants, zeta-function, Bernoulli numbers, $p$-adic measures, $p$-adic functions.

- American Mathematical Society 1978 
Our integral formula for the derivative of the regularized log gamma function (see (8) below) is an example of a very general phenomenon noticed by $D$. Barsky [2]: any Krasner analytic function on $\Omega_{p}-\mathbf{Z}_{p}^{x}$ satisfying a certain growth condition is the "Cauchy transform" of some $p$-adic measure on $\mathrm{Z}_{p}^{x}$. Theorem 2.1 below shows that, in the case of the regularized $D$-log gamma function, this measure is $\mu_{\alpha}$.

We shall also express Diamond's generalized $p$-adic Euler constants

$$
\begin{aligned}
\gamma_{p}\left(a, p^{m}\right) & =-\lim _{k \rightarrow \infty} p^{-k} \sum_{0<n<p^{k}, n \equiv a\left(\bmod p^{m}\right)} \log _{p} n \text { if } p \nmid a, \\
\gamma_{p} & =\gamma_{p}(0,1)=\frac{p}{p-1} \sum_{a=1}^{p-1} \gamma_{p}(a, p)
\end{aligned}
$$

as integrals.

2. Regularized log gamma function. Among the $p$-adic analogues of classical formulas which Diamond [3] derives for $G_{p}$ is the " $p$-adic Stirling series"

$$
G_{p}(x)=\left(x-\frac{1}{2}\right) \log _{p} x-x+\sum_{r=1}^{\infty} \frac{B_{r+1}}{r(r+1) x^{r}} \quad \text { for }|x|_{p}>1 \text {, }
$$

where $B_{k}$ is the $k$ th Bernoulli number.

Let $l(x)=\left(x-\frac{1}{2}\right) \log _{p} x-x$. Define operators $T_{p}, T_{\alpha}$ for $0 \neq \alpha \in \Omega_{p}$, by

$$
T_{p} f(x)=f(x / p), \quad T_{\alpha} f(x)=\alpha^{-1} f(\alpha x) .
$$

Then

$$
\begin{gathered}
\left(1-T_{p}\right) G_{p}(x)=G_{p}^{*}(x) \text { for } x \in \Omega_{p}-\mathbf{Z}_{p}, \\
\left(1-T_{\alpha}\right)\left(1-T_{p}\right) l(x)=-(1-1 / p) x \log _{p} \alpha,
\end{gathered}
$$

and, if we let $D=d / d x$,

$$
D T_{\alpha}=\alpha T_{\alpha} D, \quad D T_{p}=p^{-1} T_{p} D .
$$

Let $A_{r}=\left\{x \in \Omega_{p}|| x-\left.a\right|_{p}>r\right.$ for all $\left.a \in \mathbf{Z}_{p}^{x}\right\}$. Thus, $A_{1}=\left\{x \in \Omega_{p} \mid\right.$ $\left.|x|_{p}>1\right\}$. Choose $\alpha \in 1+p \mathrm{Z}, \alpha \neq 1$. Define $G_{p, \alpha}$ on $A_{1}$ by

$$
G_{p, \alpha}=\left(1-T_{\alpha}\right)\left(1-T_{p}\right)\left(G_{p}-l\right)
$$

i.e., by (3) and (4),

$$
G_{p, \alpha}(x)=\left(1-T_{\alpha}\right) G_{p}^{*}(x)+(1-1 / p) x \log _{p} \alpha \quad \text { for } x \in A_{1} .
$$

TheOREM 2.1. For $x \in A_{1}$, 


$$
\begin{gathered}
G_{p, \alpha}(x)=-\int_{\mathbf{Z}_{p}^{x}} \log _{p}(x-t) \mu_{\alpha}(t) ; \\
D^{r} G_{p, \alpha}(x)=(-1)^{r}(r-1) ! \int_{\mathbf{Z}_{p}^{x}} \frac{\mu_{\alpha}(t)}{(x-t)^{r}} \text { for } r \geqslant 1 \\
=\left(1-\alpha^{r} T_{\alpha}\right)\left(1-p^{-r} T_{p}\right) G_{p}^{(r)}(x) \text { for } r \geqslant 2 .
\end{gathered}
$$

Proof. Using (6) and (2), we write the left side of (7) as

$$
\sum_{r=1}^{\infty} \frac{1}{r x^{r}}\left(\alpha^{-r-1}-1\right)\left(1-p^{r}\right)\left(-\frac{B_{r+1}}{r+1}\right)=\sum_{r=1}^{\infty} \frac{1}{r x^{r}} \int_{\mathbf{Z}_{p}^{x}} t^{r} \mu_{\alpha}(t),
$$

by the fundamental property of $\mu_{\alpha}$, which allows it to be used to interpolate $\zeta(-r)[9]$. Hence

$$
\begin{aligned}
G_{p, \alpha}(x) & =\int_{\mathbf{Z}_{p}^{x}} \sum_{r=1}^{\infty} \frac{(t / x)^{r}}{r} \mu_{\alpha}(t)=-\int_{\mathbf{Z}_{p}^{x}} \log _{p}(1-t / x) \mu_{\alpha}(t) \\
& =-\int_{\mathbf{Z}_{p}^{x}} \log _{p}(x-t) \mu_{\alpha}(t),
\end{aligned}
$$

since $\mu_{\alpha}\left(\mathbf{Z}_{p}^{x}\right)=0$. The formula for $D^{r} G_{p, \alpha}$ now follows immediately. Q.E.D.

Corollary 2.2. For $x \in A_{1}$,

$$
\begin{aligned}
G_{p}^{* \prime}(x)-G_{p}^{* \prime}(\alpha x) & =-(1-1 / p) \log _{p} \alpha-\int_{\mathbf{Z}_{p}^{x}} \frac{\mu_{\alpha}(t)}{x-t} \\
& =-\int_{\mathbf{Z}_{p}^{x}}\left(\frac{1}{t}+\frac{1}{x-t}\right) \mu_{\alpha}(t) .
\end{aligned}
$$

The first equality in the corollary follows from (4) and (8), and the second follows from formula (12) in $\$ 4$ below.

We now use (7) to define $G_{p, \alpha}$ for $x \in \Omega_{p}-\mathbf{Z}_{p}^{x}$. This integral exists for all such $x$, since with $x \notin \mathbf{Z}_{p}^{x}$ fixed, $\log _{p}(x-t)$ is continuous in $t \in \mathbf{Z}_{p}^{x}$.

COROllary 2.3. With $G_{p, \alpha}$ defined by (7),

$$
G_{p, \alpha}(0)=\left(1-\alpha^{-1}\right) L_{p}^{\prime}(0, \omega)
$$

where $L_{p}$ is the p-adic L-function [5] and $\omega$ is the Teichmüller character.

In fact, the right-hand side equals

$$
\begin{aligned}
\lim _{k \rightarrow \infty} & \frac{1-\alpha^{-1}}{-(p-1) p^{k}} L_{p}\left(-(p-1) p^{k}, \omega\right) \\
= & -\lim _{k \rightarrow \infty} \frac{1-\alpha^{-1}}{(p-1) p^{k}} \frac{1}{1-\alpha^{-(p-1) p^{k}-1}} \\
& \cdot \int_{\mathbf{Z}_{p}^{x}} \exp \left\{(p-1) p^{k} \log _{p} t\right\} \mu_{\alpha}(t) \\
= & -\int_{\mathbf{Z}_{p}^{x}} \log _{p} t \mu_{\alpha}(t)=G_{p, \alpha}(0) .
\end{aligned}
$$


3. Analytic continuation. Diamond [3] proved that $G_{p}^{\prime \prime}=D^{2} G_{p}$ is analytic in the sense of Krasner [7] on $\Omega_{p}-Z_{p}$, but he noted that $G_{p}$ and $G_{p}^{\prime}$ are not. However, for our regularized $G_{p, \alpha}$ already the first derivative is Krasner analytic.

THEOREM 3.1. $D G_{p, \alpha}$ is Krasner analytic on $\Omega_{p}-\mathbf{Z}_{p}^{x}$.

Proof. Since $\Omega_{p}-\mathbf{Z}_{p}^{x}=\cup_{m=0}^{\infty} A_{p^{-m}}$, it suffices to show that for $m$ fixed, $f(x)=-D G_{p, \alpha}(x)$ is a uniform limit of rational functions on $A_{p-m}$ without poles there. Since

$$
f=\sum_{0<a<p^{m+1}, p \nmid a} f_{a}, \quad \text { where } f_{a}(x)=\int_{a+p^{m+1} \mathbf{Z}_{p}} \frac{\mu_{\alpha}(t)}{x-t},
$$

it suffices to show this for $f_{a}$. For $t=a+p^{m+1} s, s \in \mathbf{Z}_{p}$, we have

so that

$$
\frac{1}{x-t}=\frac{1}{x-a} \sum_{j=0}^{\infty}\left(\frac{p^{m}}{x-a}\right)^{j} p^{j_{s}^{j}}
$$

$$
f_{a}(x)=\frac{1}{x-a} \sum_{j=0}^{\infty}\left(\frac{p^{m}}{x-a}\right)^{j} p^{j} \int_{\mathbf{Z}_{p}} s^{j} \mu_{\alpha}\left(a+p^{m+1} s\right) .
$$

Since $\left|p^{m} /(x-a)\right|_{p}<1$ on $A_{p^{-m}}$ and $\left|\int_{\mathbf{z}_{\rho}}\right|_{p} \leqslant 1$, it follows that $f_{a}$ is in fact a uniform limit of rational functions on $A_{p}-m$. Q.E.D.

CoRollary 3.2. For all $x \in \Omega_{p}-\mathbf{Z}_{p}^{x}$ and all $r \geqslant 2$,

$$
\left(1-\alpha^{r} T_{\alpha}\right) G_{p}^{*(r)}(x)=(-1)^{r}(r-1) ! \int_{\mathbf{Z}_{p}^{x}} \frac{\mu_{\alpha}(t)}{(x-t)^{r}} .
$$

In fact, Diamond's argument proving Krasner analyticity of $G_{p}^{\prime \prime}$ on $\Omega_{p}$ $\mathbf{Z}_{p}$ will also prove Krasner analyticity of $G_{p}^{* \prime \prime}$ and all higher derivatives on $\Omega_{p}-\mathbf{Z}_{p}^{x}$. Since both sides of the equality are Krasner analytic on $\Omega_{p}-\mathbf{Z}_{p}^{x}$ and agree on $A_{1}$, they must be equal on all of $\Omega_{p}-\mathbf{Z}_{p}^{x}$.

COROLLARY 3.3 (SEe [4]). $D^{r} G_{p}^{*}(0)=-(r-1) ! L_{p}\left(r, \omega^{1-r}\right)$ for $r \geqslant 2$.

In fact, by Corollary 3.2, the left side equals

$$
-\left(\alpha^{r-1}-1\right)^{-1}(r-1) ! \int_{\mathbf{Z}_{p}^{x}} t^{-r} \mu_{\alpha}(t)=-(r-1) ! L_{p}\left(r, \omega^{1-r}\right) .
$$

Questions. 1. In [4, Propositions 4 and 5], Diamond proved the following relation for $L_{p}\left(r, \chi \omega^{1-r}\right)$ when $\chi$ is a Dirichlet character $\bmod p^{m}, m>1$, $r \geqslant 2$ :

$$
L_{p}\left(r, \chi \omega^{1-r}\right)=\frac{(-1)^{r}}{p^{m r}(r-1) !} \sum_{0<a<p^{m}, p \nmid a} \chi(a) D^{r} G_{p}\left(a / p^{m}\right) .
$$


Can this expression be derived using the integral formulas? The difficulty comes when trying to express $D^{r} G_{p}\left(a / p^{m}\right)$ in terms of $D^{r} G_{p, \alpha}$ when $a \neq 0$.

2. Does Corollary 3.2 hold when $r=0,1$, i.e., do we have

$$
\begin{gathered}
G_{p}^{*}(x)-\alpha^{-1} G_{p}^{*}(\alpha x)=-(1-1 / p) x \log _{p} \alpha-\int_{\mathbf{Z}_{p}^{x}} \log _{p}(x-t) \mu_{\alpha}(t), \\
G_{p}^{* \prime}(x)-G_{p}^{* \prime}(\alpha x)=-\int_{\mathbf{Z}_{p}^{x}}\left(\frac{1}{t}+\frac{1}{x-t}\right) \mu_{\alpha}(t)
\end{gathered}
$$

for all $x \in \Omega_{p}-\mathbf{Z}_{p}^{x}$ ? Is $G_{p}^{* \prime}(x)-G_{p}^{* \prime}(\alpha x)$ Krasner analytic on $\Omega_{p}-\mathbf{Z}_{p}^{x}$ ? In particular, can Corollary 2.3 be rewritten simply: $G_{p}^{*}(0)=L_{p}^{\prime}(0, \omega)$ ? Note that when $x \in p \mathbf{Z}$, the left side of (10) can be rewritten

$$
\lim _{k \rightarrow \infty} p^{-k} \sum_{0<n<p^{k}, p \nmid n} \log _{p}(n+x)-\log _{p}(n+\alpha x)=-\sum_{|x|<n<|\alpha x|, p \nmid n} \frac{1}{n}
$$

(here || means ordinary archimedean absolute value of an integer).

We obtain a partial affirmative answer to the second question in the following

THEOREM 3.4. $G_{p}^{* \prime}(x)-G_{p}^{* \prime}(\alpha x)$ is Krasner analytic on $A_{|\alpha-1|_{p}}$, and for $x \in A_{|\alpha-1|_{p}}$,

$$
\begin{aligned}
G_{p}^{* \prime}(x)-G_{p}^{* \prime}(\alpha x) & =-(1-1 / p) \log _{p} \alpha-\int_{\mathbf{Z}_{p}^{x}} \frac{\mu_{\alpha}(t)}{x-t} \\
& =-\int_{\mathbf{Z}_{p}^{x}}\left(\frac{1}{t}+\frac{1}{x-t}\right) \mu_{\alpha}(t) .
\end{aligned}
$$

Proof. Since $A_{|\alpha-1|_{p}}=\cup_{r>|\alpha-1|_{p}} A_{r}$, it suffices to write $f(x)=G_{p}^{* \prime}(x)-$ $G_{p}^{* \prime}(\alpha x)$ as a uniform limit of rational functions on $A_{r}$ for fixed $r>|\alpha-1|_{p}$. We have

$$
\begin{aligned}
f(x) & =\lim _{k \rightarrow \infty} p^{-k} \sum_{0<n<p^{k}, p \nmid n} \log _{p}(x+n)-\log _{p}(\alpha x+n) \\
& =-(1-1 / p) \log _{p} \alpha-\lim _{k \rightarrow \infty} p^{-k} \sum_{0<n<p^{k}, p \nmid n} \log _{p} \frac{x+n / \alpha}{x+n} .
\end{aligned}
$$

Thus, if we let $\alpha^{\prime}=1-1 / \alpha$ and $f_{n}(x)=\log _{p}\left(1-\alpha^{\prime} n /(x+n)\right)$, we have

$$
f(x)=-(1-1 / p) \log _{p} \alpha-\lim _{k \rightarrow \infty} p^{-k} \sum_{0<n<p^{k}, p \nmid n} f_{n}(x),
$$

where the limit is uniform on $A_{r}$. But, since $\left|\alpha^{\prime} n /(x+n)\right|_{p}<|\alpha-1|_{p} / r<1$ for $x \in A_{r}$, it follows that each

$$
f_{n}(x)=-\sum_{j=1}^{\infty} \frac{1}{j}\left(\frac{\alpha^{\prime} n}{x+n}\right)^{j}
$$


is a uniform limit of rational functions. Q.E.D.

Corollary 3.5. For $|x|_{p}<1$,

$$
\begin{aligned}
G_{p}^{* \prime}(x)-G_{p}^{* \prime}(\alpha x) & =\int_{\mathbf{Z}_{p}^{x}} \sum_{j=1}^{\infty} \frac{x^{j}}{t^{j+1}} \mu_{\alpha}(t) \\
& =\sum_{j=1}^{\infty}\left(1-\alpha^{j}\right)\left(1-p^{-j-1}\right) L_{p}\left(j+1, \omega^{-j}\right) x^{j} .
\end{aligned}
$$

4. Euler constants. In [3] Diamond defined generalized $p$-adic Euler constants by (1) above and proved that

$$
\frac{1}{p^{m-1}(p-1)} \sum_{\chi \neq \chi_{0}} \bar{\chi}(a) L_{p}(1, \chi)=\gamma_{p}\left(a, p^{m}\right)-p^{-m} \gamma_{p} \text { for } p \nmid a \text {. }
$$

Once we express $\gamma_{p}$ in terms of the $p$-adic zeta-function $\zeta_{p}$-actually, it will equal the Euler constant one would expect from a zeta-function-we can express $\gamma_{p}$ and, hence, $\gamma_{p}\left(a, p^{m}\right)$ as integrals.

Let

$$
\begin{aligned}
\tilde{\gamma}_{p} & =\frac{p}{p-1} \lim _{\varepsilon \rightarrow 0}\left(\zeta_{p}(1+\varepsilon)-(1-1 / p) / \varepsilon\right) \\
& =\frac{p}{p-1} \lim _{N \rightarrow \infty}\left(\zeta_{p}\left(1-(p-1) p^{N}\right)+(1-1 / p) /(p-1) p^{N}\right) \\
& =\frac{p}{p-1} \lim _{N \rightarrow \infty}\left[\left(1-p^{(p-1) p^{N}-1}\right)\left(-\frac{B_{(p-1) p^{N}}}{(p-1) p^{N}}\right)+p^{-N-1}\right] \\
& =-\frac{p}{p-1} \lim _{N \rightarrow \infty}\left(\frac{B_{(p-1) p^{N}}}{(p-1) p^{N}}-p^{-N-1}\right) .
\end{aligned}
$$

We claim that $\tilde{\gamma}_{p}=\gamma_{p}$. In fact, Kubota and Leopoldt $[8, \S 3]$ prove that if $A(u)=\sum_{n=0}^{\infty} a_{n}(u-1)^{n}$ converges for $|u-1|_{p}<1 / p\left(<\frac{1}{4}\right.$ if $\left.p=2\right)$, and if we let

$$
\begin{aligned}
& M^{k}(A) \underset{\text { def }}{=} p^{-k} \sum_{\substack{0<i<p^{k} \\
p \nmid i}} A(i / \omega(i)), \\
& M(A)=\lim _{k \rightarrow \infty} M^{k}(A), \quad L(u)=\sum_{n=1}^{\infty}(-1)^{n-1}(u-1)^{n} / n,
\end{aligned}
$$

then

$$
\begin{aligned}
\zeta_{p}(s) & =\frac{1}{s-1} \sum_{n=0}^{\infty} \frac{M\left(L^{n}\right)}{n !}(1-s)^{n} \\
& =\frac{1}{s-1}\left(1-\frac{1}{p}\right)+\frac{1}{s-1} \sum_{n=1}^{\infty} \frac{M\left(L^{n}\right)}{n !}(1-s)^{n} .
\end{aligned}
$$


Using this, we have

$$
\begin{gathered}
\left(1-\frac{1}{p}\right) \tilde{\gamma}_{p}=\lim _{\varepsilon \rightarrow 0}\left(\zeta_{p}(1+\varepsilon)-\left(1-\frac{1}{p}\right) / \varepsilon\right)=\lim _{\varepsilon \rightarrow 0} \frac{1}{\varepsilon} \sum_{n=1}^{\infty} \frac{M\left(L^{n}\right)}{n !}(-\varepsilon)^{\prime} \\
=-M(L)=-\lim _{k \rightarrow \infty} p^{-k} \sum_{\substack{0<i<p^{k} \\
p \nmid i}} \log _{p} i=\left(1-\frac{1}{p}\right) \gamma_{p} .
\end{gathered}
$$

This proves the claim. (The above proof, which is shorter and neater than my original proof, was kindly given me by the referee.)

Thus, Diamond's $p$-adic Euler constant agrees with the one from the Kubota-Leopoldt zeta-function.

We now derive integral formulas for $\gamma_{p}$ and $\gamma_{p}\left(a, p^{m}\right)$. In what follows we now suppose $\alpha \in 1+p^{m} Z, \alpha \neq 1$, and $\chi$ is a Dirichlet character mod $p^{m}$. For small $s$ we use

$$
\begin{aligned}
L_{p}(1+s, \chi) & =\left(\alpha^{s}-1\right)^{-1} \int_{\mathbf{Z}_{p}^{x}} t^{-s-1} \omega^{s}(t) \chi(t) \mu_{\alpha}(t) \\
& =\left(\alpha^{s}-1\right)^{-1} \int_{\mathbf{Z}_{p}^{x}} \exp \left\{-s \log _{p} t\right\} \frac{\chi(t)}{t} \mu_{\alpha}(t) .
\end{aligned}
$$

Note that $\left(\alpha^{s}-1\right) / s \rightarrow \log _{p} \alpha$ as $s \rightarrow 0$. Let $\varepsilon(\chi)=1$ if $\chi=\chi_{0}, 0$ otherwise. Then

$$
\frac{1}{\log _{p} \alpha} \int_{\mathbf{Z}_{p}^{x}} \frac{\chi(t)}{t} \mu_{\alpha}(t)=\lim _{s \rightarrow 0} s L_{p}(1+s, \chi)=(1-1 / p) \varepsilon(\chi) .
$$

Define $\gamma_{p}(x)$ by

Then

$$
(1-1 / p) \gamma_{p}(\chi)=\left\{\begin{array}{l}
\lim _{s \rightarrow 0}\left(L_{p}(1+s, \chi)-\frac{1-1 / p}{s} \varepsilon(\chi)\right) \\
(1-1 / p) \gamma_{p} \text { if } \chi=\chi_{0}, \\
L_{p}(1, \chi) \text { otherwise. }
\end{array}\right.
$$

$$
\begin{aligned}
(1-1 / p) \gamma_{p}(\chi) & =\lim _{s \rightarrow 0} \int_{\mathbf{Z}_{p}^{x}} \frac{1}{\alpha^{s}-1} \frac{e^{-s \log _{p} t} \chi(t)}{t}-\frac{\chi(t)}{s t \log _{p} \alpha} \mu_{\alpha}(t) \\
& =\lim _{s \rightarrow 0} \int_{\mathbf{Z}_{p}^{x}}\left(\frac{e^{-s \log _{p} t}}{e^{s \log _{p} \alpha}-1}-\frac{1}{s \log _{p} \alpha}\right) \frac{\chi(t)}{t} \mu_{\alpha}(t) \\
& =\lim _{s \rightarrow 0} \frac{1}{s \log _{p} \alpha} \int_{\mathbf{Z}_{p}^{x}}\left(\frac{1-s \log _{p} t}{1+(s / 2) \log _{p} \alpha}-1\right) \frac{\chi(t)}{t} \mu_{\alpha}(t) \\
& =-\int_{\mathbf{Z}_{p}^{x}}\left(\frac{\log _{p} t}{\log _{p} \alpha}+\frac{1}{2}\right) \frac{\chi(t)}{t} \mu_{\alpha}(t) .
\end{aligned}
$$


Since (11) gives us

$$
\begin{aligned}
(1-1 / p) \gamma_{p}\left(a, p^{m}\right) & =p^{-m}\left((1-1 / p) \gamma_{p}+\sum_{\chi \neq \chi_{0}} \bar{\chi}(a) L_{p}(1, \chi)\right) \\
& =p^{-m}(1-1 / p) \sum_{\text {all } \chi} \bar{\chi}(a) \gamma_{p}(\chi) \\
& =-p^{-m} \int_{\mathbf{Z}_{p}^{x}}\left(\frac{\log _{p} t}{\log _{p} \alpha}+\frac{1}{2}\right) \frac{1}{t} \sum_{\chi} \chi(t / a) \mu_{\alpha}(t),
\end{aligned}
$$

we may conclude

THEOREM 4.1 .

$$
(1-1 / p) \gamma_{p}\left(a, p^{m}\right)=-\int_{a+p^{m} \mathbf{Z}_{p}}\left(\frac{\log _{p} t}{\log _{p} \alpha}+\frac{1}{2}\right) \frac{\mu_{\alpha}(t)}{t} .
$$

REMARK. Note that

$$
G_{p}^{\prime}\left(a / p^{m}\right)=\lim _{k \rightarrow \infty} p^{-k} \sum_{0<n<p^{k}} \log _{p}\left(\frac{a}{p^{m}}+n\right)=-p^{m} \gamma_{p}\left(a, p^{m}\right),
$$

and, similarly,

$$
G_{p}^{* \prime}(0)=-(1-1 / p) \gamma_{p}
$$

Using Diamond's relation (9) in the same way as we used (11) to prove Theorem 4.1, we see that for $r \geqslant 2$,

$$
G_{p}^{(r)}\left(a / p^{m}\right)=p^{m r}(r-1) !(-1)^{r}\left(\alpha^{r-1}-1\right)^{-1} \int_{a+p^{m} \mathbf{Z}_{p}} \frac{\mu_{\alpha}(t)}{t^{r}} .
$$

Namely, replace the left-hand side of (9) by $\left(\alpha^{r-1}-1\right)^{-1} \int_{\mathbf{z}_{p}^{\times}} \chi(t) t^{-r} \mu_{\alpha}(t)$. Then let $\chi$ run over all characters $\bmod p^{m}$, for each $\chi$ multiply $(9)$ by $\bar{\chi}(a)$, and take the sum. One obtains

$$
\begin{aligned}
\left(p^{m}-p^{m-1}\right) & \left(\alpha^{r-1}-1\right)^{-1} \int_{a+p^{m} \mathbf{Z}_{p}} t^{-r} \mu_{\alpha}(t) \\
= & \frac{(-1)^{r}}{p^{m r}(r-1) !}\left(p^{m}-p^{m-1}\right) D^{r} G_{p}\left(a / p^{m}\right) .
\end{aligned}
$$

ACKNowledgment. I would like to thank B. Mazur, D. Kazhdan,and N. Katz for helpful discussions. I would also like to thank Ju. I. Manin and D. Leščiner for showing me the integral formula for the Kubota-Leopoldt $\gamma_{p}$, on which $\$ 4$ is based.

\section{REFERENCES}

1. E. Artin, The gamma function, Holt, Rinehart and Winston, New York, 1964.

2. D. Barsky, L'algèbre d'Iwasawa (to appear). 
3. J. Diamond, The p-adic log gamma function and p-adic Euler constants, Trans. Amer. Math. Soc. 233 (1977), 321-337.

4. $\ldots$ On the values of $p$-adic L-functions at positive integers (to appear).

5. K. Iwasawa, Lectures on p-adic L-functions, Princeton Univ. Press, Princeton, N. J., 1972.

6. N. Koblitz, p-adic numbers, $p$-adic analysis, and zeta-functions, Graduate Texts in Math., No. 58, Springer, New York, 1977.

7. M. Krasner, Rapport sur le prolongement analytique dans les corps valués complets par la méthode des éléments analytiques quasi-connexes, Table Ronde d'Analyse Non Archimédienne (Paris, 1972), Bull. Soc. Math. France, Mém. No. 39-40, Soc. Math. France, Paris, 1974, pp. 131-254.

8. T. Kubota and H. Leopòldt, Eine p-adische Theorie der Zetawerte. I, J. Reine Angew. Math. 214/215 (1965), 328-339.

9. B. Mazur, Analyse p-adique, special Bourbaki redaction.

Department of Mathematics, Harvard University, Cambridge, Massachusetts 02138 